Aus dem Institut für experimentelle Forschung (Leiter: Prof. B. Nyg a ard-Ostbye), Odontologische Fakultät, Universität Oslo und Center of Human Growth and Development (Leiter: Prof. R. E. Moyers) Craniofacial Program (Leiter: Prof. D. H. Enlow) University of Michigan, Ann Arbor ${ }^{1}$

\title{
Die gewebliche Übertragung mechanisch induzierter Kräfte vom Gebiß auf das Gesicht
}

\author{
Von L. Linge, Skien (Norwegen)
}

Mit 1 Abbildung

In der praktischen Kieferorthopädie spielen mechanisch induzierte Kräfte eine bedeutende Rolle [23, 27]. Sie stehen uns in einer reichen Auswabl zur Verfügung; ihre Verwertbarkeit ist aber stark von Kenntnissen, Zielsetzung und praktischer Fahigkeit des jeweiligen Kieferorthopäden abhängig.

Aus der Physik wissen wir, daß eine mechanisch winksame Kraft immer von einer gleich großen, entgegengerichteten Kraft gefolgt wird: Aktion ist gleich Reaktion. Ein entscheidender Unterschied liegt jedoch zwischen Mechanik im Sinne der Physik und Mechanik im Sinne der Kieferorthopädie vor. Durch die Qualität des kraftauffangenden Mediums bedingt, läuft die biologische Reaktion anders als die mechanische des toten Untersuchungsobjektes ab $[4,11,15]$.

Aus der Basalforschung und aus der klinischen Forschung, vor allem im Bereich des Parodontes, sind schon eine Reihe von Zusammenhänge zwischen mechanisch induzierten Kräften und den dadurch ausgelösten Gewebsreaktionen. bekannt $[22,28,29]$. Es besteht zur Zeit noch eine Lücke im Wissen über die zu erwartenden Gewebsreaktionen in den Wachstumszonen des Gesichtsschädels unter Beeinflussung der kieferorthopädischen Therapie. Teilweise mag dies daran liegen, daß eine exakte Definition der beiden Komponenten, der wirksamen Kraft und des kraftauffangenden Gewebes, außerordentlich problematisch ist. Hinzu kommt die große Schwierigkeit der Objektivierung bei solchen Untersuchungen $[1,2,3,14]$.

Die Kieferorthopäden haben seit Jahren den Ehrgeiz gehabt, nicht allein die Zähne zu richten, sondern zusätzlich maßgebenden FinfluB auf die gesamte Gesichtsentwicklung zu üben $[9,16,17,26]$. In der Praxis ist das Richten der Zähne allerdings die erste Aufgabe geblieben, wobei andere und erweiterte Behandlungsziele in den letzten Jahren immer mehr in den Vordergrund getreten sind $[13,25]$.

${ }^{1}$ Diese Arbeit wurde teilweise von U.S.P.H.S., Grant 02272 unterstuitzt. 
In einer vielseitigen Praxis von heute kann man die Sachgebiete folgender. maßen einteilen:
a) Orthodontie.
b) Myo-funktionelle Therapie.
c) Gesichtsorthopädie.
d) Kieferorthopädische Chirurgie

In der Orthodontie setzt man sich das Ziel, die Zähe innerhalb des Kieferfortsatzes, durch von unseren therapeutischen Maßnahmen hervorgerufene Um. bauten im Bereich des Parodontes, zu richten. Dabei legt man sich nicht auf ein bestimmtes Behandlungsverfahren fest.

In der myo-funktionellen Therapie sucht man in der ersten Beihe neuromuskuläre Funktionen zu lenken und gegebenenfalls umzugestalten. Hier kommen ebenfalls verschiedenartige Behandlungsmittel in Frage.

In der Gesichtsor thopädie verwendet man bewußt therapeutische Kräfte, die stark genug sind, um die Resistenz des kraftauffangenden Gewebes teilweise oder ganz zu überwinden, um dadurch eine relative Verschiebung der Bauelemente des Gesichtsschädels zu erreichen. Diese Behandlungsmethode setzt Geräte voraus, die eine besonders straffe Lenkung der Kraft ermöglichen.

Die kieferorthopädische Chirurgie wird von uns mitgeplant, vorbereitet und nachbehandelt. Dabei wird unser Wissen über Gesichtswachstum und Ausgleichsmöglichkeiten im Bereich des Gebisses besonders in Anspruch genommen.

Auf allen diesen Teilgebieten unserer klinischen Arbeit sind Uberlegungen bezüglich der mechanisch induzierten Kräfte eine wichtige Voraussetzung. Die von uns geübte Kraft, ab jetzt als Primärkraft bezeichnet, ist die erste Stufe einer langen Reihe von sekundär entstehenden Kräften, die in den einzelnen Gewebsabschnitten die eigentliche Gewebsreaktion auslösen ${ }^{1}$.

Klinisch ist die Primärkraft sehr von Aufbau and Applikation unseres Geräts abhängig, wobei die Art der Verankerung und die Lage des Angriffspunktes im Verbältnis zux Anatomie und zur geweblichen Fixierung des Gebiß bzw. Gesichts: teils ausschlaggebend ist. Unser Verankerungssystem wird im Rahmen der gezielten Lenkung während des Behandlungsablaufs, je nach Art der wirksamen Kräfte, unterschiedlich in Anspruch genommen. Die Beanspruchung ist weiterhin von der Gewebsreaktion und den wechselnden anatomischen Gegebenheiten abhängig.

Die entstandenen Sekundärkräfte sind, je weiter man von dem Angriffspunkt im Bereich des Gebisses in die tieferen Strukturen des Gesichts hineinkommt, zunehmend schwerer zu definieren, sowohl in bezug auf Richtung als auch auf Intensität. Sie sind weitgehend von örtlicher Anatomie, mechanischen Eigenschaften des Gewebes und Gewebsreaktionen abhängig.

Die aktivierten Kräfte werden von allen Gewebskomponenten des betreffenden Gesichtsbezirkes aufgefangen - nicht allein vom Stützgewebe. Die erreichte morphologische Veränderung ist ein Ergebnis der totalen, vitalen Aktivität sämtlicher Gewebsbestandteile, wobei allerdings nicht alle gleichermaßen und direkt morphogenetisch aktiv sind.

Von dem Wirkungsweg der therapeutischen Kräfte könnte man in großen Zügen sagen: er läuft von den Zähnen über die Parodontalfasern zum Kiefer-

1 In dem Vortrag wurde an dieser Stelle die Wirkung der Primärkraft und die dadurch ausgelösten Sekundärkräfte an Hand eines klinischen Beispiels, aus dem Bereich der Gesichtsorthopädie, mittels Lichtbildern und Fernröntgenaufnahmen dargestellt. 
knochen, um über die Fasern der Suturen zu den umgebenden Gesichtsknochen. zu gelangen [12]. Uber den Gesichtsschädel hinaus wird er sich nur in Ausnahmefällen folgen lassen [10].

Um die jeweiligen Gewebsreaktionen systematisch zu studieren, sind aber eine Reihe von wichtigen Umständen zu berücksichtigen, die Kenntnisse der ablaufenden Gewebsaktivität in dem entsprechenden Gebiet voraussetzen $[5,6,7,8,18$, 20, 21]. Die primären Kräfte und die sekundär entstehenden Kräfte werden den Gewebsaktivitäten aufgepfropft, die schon von physiologischen, genetischen und wachstumsbedingten Faktoren gelenkt sind. Die kieferorthopädische Behandlung besteht aus einer langen Serie von aufeinander folgenden und teilweise überlager ten Gewebsreaktionen, die der normalen Gewebsaktivität beigefügt sind. Um unsere klinischen Methoden zu verbessern, benötigen wir alle uns zugängigen $\mathrm{Er}$ kenntnisse auf diesem Gebiet.

Für die praktische Forschung bietet sich die tierexperimentelle Untersuchung an, wobei allerdings die komplexen Fragen zergliedert werden müssen - und die biologischen Unterschiede von Tier zu Mensch nicht in Vergessenheit geraten darf [24].

Die Hauptpunkte einer solchen Forschungsaufgabe können folgendermaßen dargestellt werden (Abb. 1).

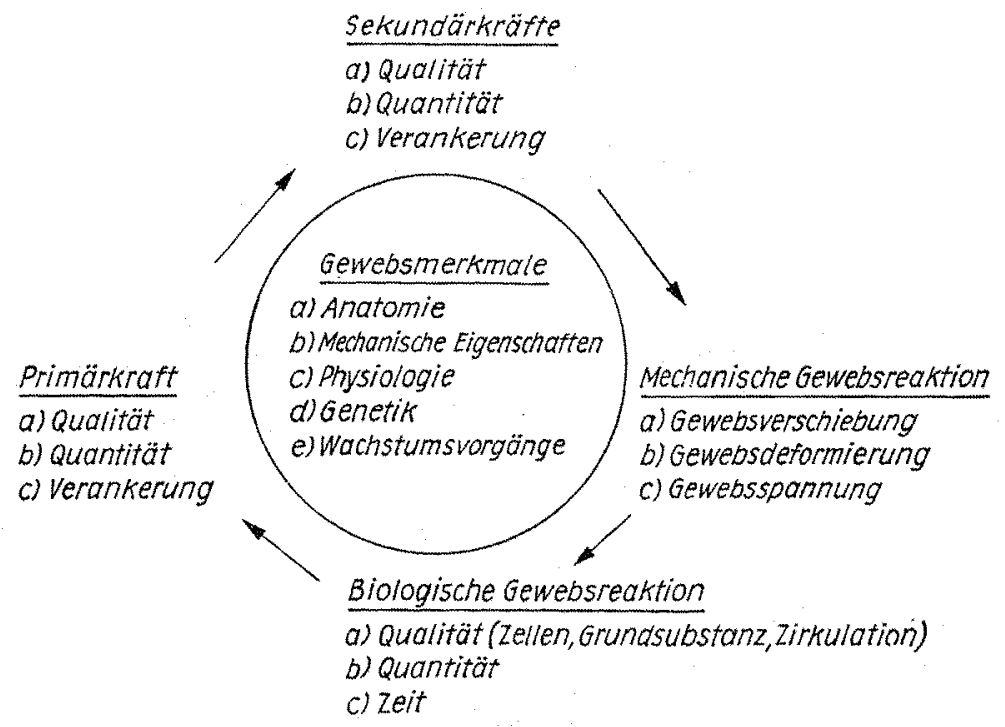

Abb. 1

Die Trennung in mechanische und biologische Gewebsreaktionen scheint aus praktischen Gründen zweckmäßig zu sein, obwohl eine absolute Abgrenzung der beiden Gebiete kaum möglich ist. Uber eine Zeitspanne wirkt sich die Gewebsreaktion morphologisch und funktionell aus, um dadurch das biologische Unter*. suchungsobjekt maBgebend zu beeinträchtigen $[15,19]$.

Der ständigen. Wechselwirkung zwischen den einzelnen Komponenten dieses Systems muß groBe Bedeutung zugemessen werden. Die von uns therapeutisch 
geübten Primärkräfte werden dadurch lediglich zu Impulsen in einem geschlossenen Ringsystem, wo es keinen Anfang und kein Ende gibt - wo man sich als Kliniker nur anschließen darf.

\section{Zusammenfassung}

Eine sachliche Gliederung der klinischen Kieferorthopädie wird vorgeschlagen. Es wird darauf hingewiesen, daß mechanisch induzierte Kräfte anf allen Teilgebieten des Faches eine bedeutende Rolle spielen.

Die von dem kieferorthopädischen Gerät entwickelte Kraft wird als Primärkraft bezeichnet, während Kräfte, die sich beim Auffangen der Primärkraft in den Geweben des Gesichtes entwickeln, Sekundärkräfte genannt werden.

Die eigentliche Gewebsreaktion wird von Sekundärkräften ausgelöst. Unterschiede und gegenseitige Beziehungen zwischen mechanische and biologische Gewebsreaktionen werden diskutiert und sehematiseh dargestellt.

\section{Summary}

The significance of force application in all aspects of clinical orthodontics is stressed. Experimental work is still needed to learn more about the effect of such forces on facial tissues. The increasing attention of modern orthodontics with attempts to influence facial growth and development makes this research also important to the clinician.

A distinetion in primary (- mechanically induced) force and secondary (- tissue related) foree is proposed. Secondary forces are strongly related to local circumstance and tissue characteristics. Tissue reactions in facial structures are mainly initiated by secondary forces.

Differences between mechanical and biological tissue reactions, as well as their interdependence is discussed.

\section{Sclirilttum}

1. Bassett, C. A., und R. O. Becker, Generation of electric potentials by bone in response to mechanical stress. Science 137 (1962) 1063. - 2. Bassett, A. I., Electrical effects in bone. Scientific Amer. 1965, 18-25. - 3. Baumrind, S., A reconsideration of the propriety of the "pressure-tension" hypothesis. Amer. J. Orthodont. 55 (1969) 12-22. 4. Bien, S. M., Fluid dynamic mechanisms which regulate tooth rovement. Advanc. oral. Biol. 2 (1966) 173-201. - 5. Bjork, A., Facial growth in man, studied with the aid of metallic implants. Acta odont. scand. 13 (1955) 9-34. - 6. Enlow, D. H., Principles of Bone Remodeling. Charles C. Thomas Publishers, Springfield/Ill. 1963. - 7. Enlow, D. H., A morphogenetic analysis of facial growth. Amer. J. Orthodont. 52 (1966) 283-299. 8. Enlow, D. H., A comparative study of facial growth in Homo and Macaca. Amer. J. phys. Anthrop. 24 (1966) 293-307. - 9. Fränkel, R., Orthodontie, Kieferorthopädie, Funktions kieferorthopädie. Dtsch. Stomat. 15 (1965) 299. - 10. Gardner, G. E., und J. H. Kronman, Cranioskeletal displacements caused by rapid palatal expansion in the rhesus monkey. Amer. J. Orthodont. 59 (1971) 146-155. - 11. Gianelly, A. A., Force-induced changes in the vascularity of the periodontal ligment. Amer. J. Orthodont. 55 (1969) 5-11. - 12. Graber, Chung, und Aoba, Dentofacial orthopedics versus orthodontics. J. Amer. dent. Ass. 75 (1967) 1145-1166. - 13. Haas, A. J., Palatal expansion: Just the beginning of dentofacial orthopedics. Amer. J. Orthodont. 57 (1970) 219-255. - 14. Is a acson, R. J., J. L. Wood und A. H. Ingram, Forces produced by rapid maxillary expansion. Angle Orthodont. 34 (1964) $256-270$. - 15. Jacobs, R. M., Treatment objectives and case retention: Cybernetic and "Myometrie" considerations. Amer. J. Orthodont. 58 (1970) 552-564. - 16. Korkkaus, G., Present orthodontic thought in Germany. Amer. J. Orthodont. 45 (1959). - 
17. Korkhaus, G., Orthodontie oder Kieferorthopädie? Zahnüxztl. Mschr. H. 1.3 (1962). 18. Linge, L., A technique for the study of morphology in facial sutures under mechanical influence. Trans. Europ. Orthodont. Soc. 1970,553-567. - 19. Melsen, B., und H. Enemark, Effect of cervical anchorage studied by the implant method. Trans. Europ. Orthodont. Soc. 1969, 435-444. - 20. Prah1, B., Sutural Growth. Diss. der Universität Nijmegen (Holland) 1968. - 21. Pritchard, Scott und Girgis, The structure and development of cranial and facial sutures. J. Anat. (Lond.) 90 (1956) 73-86. - 22. Peita $\mathrm{n}$, K., The initial tissue reaction incident to orthodontic tooth movement as related to the influence of function. Acta odont. scand., Suppl. 6 (1951). - 23. Reitan, K., Some factors determining the evaluation of forces in orthodontics. Amer. J. Orthodont. 43 (1957) 32-45. - 24. Reitan, K., und E. Kram, Comparative behavior of human and animal tissue during experimental tooth movement. Angle Orthodont. 41 (1971) 1-14. - 25. Ricketts, R. M., Planning treatment on the basis of the facial pattern and an estimate of its growth. Angle Orthodont. 27 (1957) 14-37. 26. Ricketts, R. M., The influence of orthodontic treatment on facial growth and development. Angle Orthodont. 30 (1960) 103-133. - 27. Schwarz, A. M., Die lebenskundlichen (biologischen) Grundlagen der Kieferorthopädie. In: Lehrgang der GebiBregelung, Bd. II, S. 6-72. Urban \& Schwarzenberg, Wien-Innsbruck 1956. - 28. Storey, E., und R. Smith, Force in orthodonties and its relation to tooth movement. Aust. J. Dent. 56 (1952) 11-18. - 29. Stuteville, O. H., A summary review of tissue changes incident to tooth movement. Angle Orthodont. 8 (1938) $1-20$.

Anschr. d. Verf.: Dr. Leif Linge, Institut für experimentelle Forschang, Odontologische Fakultät der Universität OSLO, Blindern (Norwegen) 